\title{
Sound-propagation gap in fluid mixtures
}

\author{
Supurna Sinha and M. Cristina Marchetti \\ Physics Department, Syracuse University, Syracuse, New York 13244
}

\begin{abstract}
We discuss the behavior of the extended sound modes of a dense binary hard-sphere mixture. In a dense simple hard-sphere fluid the Enskog theory predicts a gap in the sound propagation at large wave vectors. In a binary mixture the gap is only present for low concentrations of one of the two species. At intermediate concentrations sound modes are always propagating. This behavior is not affected by the mass difference of the two species, but it only depends on the packing fractions. The gap is absent when the packing fractions are comparable and the mixture structurally resembles a metallic glass.
\end{abstract}

\section{INTRODUCTION}

Short-wavelength collective modes in dense simple hard-sphere fluids have been studied extensively by both theoretical and experimental researchers in the past few years. ${ }^{1-4}$ Two interesting features of the extended hydrodynamic modes in a simple fluid are the softening of the heat mode at the wave vector $k$ where the static structure factor $S(k)$ has its first maximum and the appearance of a gap in the sound propagation in the same large-wave-vector region. ${ }^{1,2}$ The softening of the heat mode corresponds to the slowing down of structural relaxation in a dense fluid and has been discussed extensively in the literature. The sound-propagation gap consists in the vanishing of the imaginary part of the frequency of the sound waves, which describes the propagation of the wave, over a finite region of $k$ values.

In a one-component fluid of hard spheres of size $\sigma$ and density $n$ one obtains a gap in the sound propagation for all values of the packing fraction $\eta=(\pi / 6) n \sigma^{3}$ above $\eta \approx 0.33 .^{5}$ This feature of the extended modes has been the topic of some controversy. de Schepper, Cohen, and Zuilhof, ${ }^{5}$ have shown that the appearance and disappearance of the sound propagation gap can be understood as arising from the competition between generalized wave-vector-dependent reversible elastic restoring forces and dissipative forces in the fluid. Roughly speaking, a gap arises whenever 
the dissipation exceeds the elastic forces. They also pointed out that the behavior of the sound modes in a one-component fluid is quite insensitive to the static structure factor of the fluid and the gap is present even when setting $S(k)=1$. de Schepper, Van Rijs, and Cohen ${ }^{6}$ have also shown that even the long-wavelength Navier-Stokes equations, when used outside their range of validity to describe shortwavelength phenomena, predict a gap in the sound propagation. The model based on the Navier-Stokes equations suggests that there is no connection between local effects leading to wave-vector-dependent transport coefficients and susceptibilities and the trapping of sound on molecular length scales.

In a recent paper (hereafter denoted $\mathbf{I}$ ) we used the Enskog equation as the starting point to study the short-wavelength collective excitations of a binary hard-sphere fluid mixture. ${ }^{7}$ In a mixture the spatial ordering of the particles can be changed while keeping the total density of the fluid constant by changing the concentration $x_{2}=n_{2} /\left(n_{1}+n_{2}\right)$ of the larger species (here type- 2 spheres) or the size ratio $\alpha=\sigma_{1} / \sigma_{2}$. We find that sound propagation in the mixture depends on the concentration $x_{2}$ : the trapping of sound modes and the corresponding propagation gap that arises in dense simple fluids only occurs in mixtures with a small concentration of small spheres in a dense fluid of large spheres $\left(x_{2} \approx 1\right)$ or a small concentration of large spheres in a dense fluid of small spheres $\left(x_{2} \ll 1\right)$. At intermediate values of $x_{2}$ we observe a softening of the sound propagation, but no actual gap. For either small or large values of $x_{2}$ the mixture closely resembles a simple fluid, as can be seen from the behavior of the partial structure factors $S_{11}(k), \quad S_{22}(k)$ and $S_{12}(k)$ defined in I. For instance, for $x_{2}=0.01$ only the static structure factor $S_{11}(k)$ of the dense component is peaked [see Fig. 1(a)]. The other structure factors are essentially constant. Thus we expect the appearance of a sound-propagation gap as in a simple fluid. At intermediate values of the concentration $x_{2}$ the dense mixture resembles a metallic glass: there is short-range order in the spatial arrangements of both types of spheres, as indicated by the fact that all three static structure factors display considerable structure as functions of the wave vector [see Fig. 1(b)]. The absence of a sound-propagation gap in this case is then consistent with the experimental finding that no gap occurs in the second sound of metallic glasses. ${ }^{8}$ This observation seems to indicate that the local fluid structure does play a role in determining sound propagation and sound trapping at short wavelengths, in contrast to what was argued for a one-component fluid.

In this paper we investigate in more detail the extended sound modes in binary mixtures, with the objective of clarifying their dependence on concentration, size 
ratio and mass ratio, and the role of the fluid structure in determining the gap. As in our earlier paper, we keep the total packing fraction of the mixture $\eta=$ $(\pi / 6)\left(n_{1} \sigma_{1}^{3}+n_{2} \sigma_{2}^{3}\right)$ fixed at the value $\eta=0.46$, which is close to the packing fraction corresponding to freezing of a one-component hard-sphere fluid.

FIG.1. The partial static structure factors $S_{11}(k), S_{22}(k)$, and $S_{12}(k)$ for $\eta=0.46$, $\alpha=0.7, x_{2}=0.01(\mathrm{a})$, and $x_{2}=0.5(\mathrm{~b})$.

First we have considered the dependence of the sound modes on the ratio $m_{1} / m_{2}$ of the masses of the two species and the role of this ratio in determining the propagation gap. By comparing the extended hydrodynamic modes displayed in $\mathbf{I}(\alpha=0.7$ and $\left.m_{1} / m_{2}=0.5\right)$ to the modes obtained for the same values of size ratio and concentration, but equal masses, we have found that the sound propagation is essentially unchanged at all concentrations. This holds true for other values of the size ratio. In general, as long as the mass ratio is not too small $\left(m_{1} / m_{2} \geq 0.1\right)$ the difference in mass of the two species affects only weakly the sound propagation in a mixture. This points to the fact that the size difference and the spatial ordering of the two types of spheres must play the main role in determining the absence of sound trapping at large wave vectors.

In Sec. II we discuss the dependence of the sound modes on the concentration of one of the two species. We show that in dense mixtures, as in a one-component fluid, the appearance of a sound propagation gap can be understood as the result of the competition between elastic and dissipative forces in the fluid. The absence of the sound-propagation gap in mixtures of intermediate concentrations is not determined by the details of the static structure factors, but it does depend on the fluid structure in the sense that it occurs when the packing fractions $\eta_{1}=(\pi / 6) n_{1} \sigma_{1}^{3}$ and $\eta_{2}=(\pi / 6) n_{2} \sigma_{2}^{3}$ of the two species are comparable. In this case all three static

structure factors are peaked and the structural properties of the mixture resemble those of a metallic glass.

\section{DEPENDENCE OF SOUND PROPAGATION ON CONCENTRATION}

We showed in $\mathbf{I}$ that at large wave vectors the extended heat and diffusion modes govern the relaxation of the densities of the two species. These are the only long- 
lived fluctuations in the fluid at these wave vectors. At large wave vectors the extended sound modes mainly describe the relaxation of temperature and longitudinal momentum fluctuations. Similarly, in a one-component fluid large-wave-vector density fluctuations are long-lived while temperature and momentum fluctuations relax quickly. On the basis of this observation Zuilhof and co-workerss suggested that extended sound modes in a one-component fluid can be described by a simple model obtained by neglecting the coupling to the density in the generalized hydrodynamic equations for temperature and longitudinal momentum. The resulting two coupled equations have eigenfrequencies that closely reproduce the extended sound modes at large wave vectors. Clearly, at small wave vectors these modes do not reduce to the hydrodynamic sound modes.

The same model can be used to describe the extended sound modes of a mixture, where the characteristic time scale for the relaxation of temperature and momentum fluctuations is well separated from that governing the relaxation of fluctuations in the two densities. If we neglect the coupling to fluctuations in the densities in the generalized hydrodynamic equations for temperature and longitudinal momentum, we obtain two coupled equations that are formally identical to those obtained for a one-component fluid. The eigenvalues of these equations are obtained by solving a quadratic equation, with the result

$$
\begin{aligned}
z_{ \pm}(k)= & -\frac{1}{2}\left[\Omega_{l l}(k)-\Omega_{T T}(k)\right] \\
& \pm \frac{1}{2}\left\{\left[\Omega_{l l}(k)-\Omega_{T T}(k)\right]^{2}-4[f(k)]^{2}\right\}^{\frac{1}{2}}
\end{aligned}
$$

where $\Omega_{l l}(k)$ and $\Omega_{T T}(k)$ are the damping rates in the equations for longitudinal momentum and temperature fluctuations, respectively, and $f(k)=i \Omega_{T l}(k)$ is the elastic restoring force that couples the two equations,

$$
\begin{array}{r}
\Omega_{l l}(k)=\frac{2}{3 \rho} \sum_{a=1,2} \sum_{b=1,2} \frac{2 \mu_{a b} \sqrt{n_{a} n_{b}}}{t_{E_{a b}}}\left[1-j_{0}\left(k \sigma_{a b}\right)\right. \\
\left.+2 j_{2}\left(k \sigma_{a b}\right)\right] \\
\Omega_{T T}(k)=\frac{2}{3 \rho} \sum_{a=1,2} \sum_{b=1,2} \frac{2 \mu_{a b} \sqrt{n_{a} n_{b}}}{t_{E_{a b}}}\left[1-j_{0}\left(k \sigma_{a b}\right)\right]
\end{array}
$$




$$
\begin{array}{r}
f(k)=k \sqrt{2 n / 3 \beta \rho}\left[1+\sum_{a=1,2} \sum_{b=1,2} 2 \pi \frac{n_{a} n_{b}}{n} \sigma_{a b}^{3}\right. \\
\left.\times \chi_{a b} \frac{j_{1}\left(k \sigma_{a b}\right)}{k \sigma_{a b}}\right],
\end{array}
$$

where $n=n_{1}+n_{2}$ and $\rho=m_{1} n_{1}+m_{2} n_{2}$ are the total number and mass densities, respectively. Also, $\mu_{a b}=m_{a} m_{b} /\left(m_{a}+m_{b}\right)$ is the reduced mass, $\chi_{a b}$ the pair correlation function of species $a$ and $b$ at contact, $\sigma_{a b}=\left(\sigma_{a}+\sigma_{b}\right) / 2$, and $j_{n}(x)$ is a spherical Bessel function of order $n$. Finally, $t_{E_{a b}}$ is the Enskog mean-free time, $\left.t_{E_{a b}}=\sqrt{2 \mu_{a b} \beta} / 4 \sqrt{\pi n_{a} n_{b}} \sigma_{a b}^{2} \chi_{a b}\right)$. At large wave vectors the two modes given in Eq. (2.1) closely resemble the extended sound modes obtained in $\mathbf{I}$ by solving the four coupled hydrodynamic equations. The argument of the square root in Eq. (2.1) is generally negative (and the two modes are propagating), unless the dissipative damping exceeds the elastic forces. In this case Eq. (2.1) yields two real roots, corresponding to diffusive modes. This is the region of the soundpropagation gap. The argument of the square root in Eq. (2.1) can be factorized as $\left(\Omega_{l l}-\Omega_{T T}+2 f\right)\left(\Omega_{l l}-\Omega_{T T}-2 f\right)$. The first factor is always positive. We define the function $\Delta(k)$ by

$$
\frac{\sigma_{12}}{2}\left[\frac{3 \beta \rho}{2 n}\right]^{1 / 2}\left(\Omega_{l l}-\Omega_{T T}-2 f\right)=\Delta(k)-k \sigma_{12}
$$

with

$$
\Delta(k)=\frac{2 \pi}{n} \sum_{a=1,2} \sum_{b=1,2} n_{a} n_{b} \sigma_{a b}^{2} \sigma_{12} \chi_{a b}\left[\left[\frac{16 \mu_{a b} n}{3 \pi \rho}\right]^{1 / 2} j_{2}\left(k \sigma_{a b}\right)-j_{1}\left(k \sigma_{a b}\right)\right] .
$$

A gap occurs when $\Delta(k)>k \sigma_{12}$. This condition is displayed graphically in Fig. 2 at three different concentrations. The figure shows that a gap is obtained for $x_{2}=0.01$ and $x_{2}=0.9$, but not for $x_{2}=0.5$. For $\alpha=0.7$ and $m_{1} / m_{2}=0.5$ this simple model predicts no sound-propagation gap for $0.05 \leq 0.7$, in agreement with what was obtained in I solving the full coupled hydrodynamic equations.

FIG.2. The straight line is $k \sigma_{12}$. The curves represent the function $\Delta(k)$ for $\eta=0.46, \alpha=0.7$, and $m_{1} / m_{2}=0.5$, and $x_{2}=0.01$ (solid line), $x_{2}=0.5$ (dashed 
line), and $x_{2}=0.9$ (chain dashed line), as a function of $k \sigma_{12}$.

In mixtures, as in a one-component fluid, the sound-propagation gap occurs as a consequence of the competition between dissipative and reversible forces. In most of the wave-vector range elastic forces exceed dissipative forces. This simply reflects the fact that sound modes propagate in both liquids and low-density gases (in the very-large-wave-vector limit the fluid resembles an ideal gas, since one is considering length scales that are too short for the interactions to be relevant). The damping term and the elastic forces depend on the packing fractions $\eta_{1}$ and $\eta_{2}$. For a fixed total packing fraction $\eta$ and size ratio $(\alpha=0.7)$, both dissipative and elastic terms decrease with increasing $x_{2}$. At all $x_{2}$ there is a region of large $k$ where the dissipation rates are essentially constant (and equal the sum of the Enskog times weighted by the mass fractions) and $\Omega_{l l}-\Omega_{T T} \approx 0$. For $x_{2} \ll 1$ and $x_{2} \simeq$ the elastic forces vanish even more rapidly than the dissipative term in this large- $k$ region and a gap occurs. For intermediate $x_{2}$ the elastic forces remain finite at all $k$ and the modes are always propagating.

In a recent paper ${ }^{9}$ Campa and Cohen suggested that the static structure factors may play a role in determining sound-propagation gaps in a mixture. On the other hand, the simple model described above does not contain the static structure factors $S_{i j}(k)$. In fact, the appearance and disappearance of the sound-propagating gap are insensitive to the structure factors. This can also be seen by reconsidering the extended sound modes obtained in I from the solution of the four coupled hydrodynamic equation and arbitrarily setting $S_{11}(k)=S_{22}(k)-1$ and $S_{12}(k)=0$ in these equations. One finds that, while the gap itself, when it occurs, is wider if the proper static structure factors are included, its concentration dependence, i.e., its appearance and disappearance, is qualitatively unchanged. The suggestion of Campa and Cohen is, however, in the right direction and it would be misleading to say that short-wavelength sound modes in a mixture are not affected by the fluid structure. The latter does enter even in the simple model yielding Eq. (2.1) through the dependence on the packing fractions of the two components of the mixture. When the packing fractions are both appreciable, there is not gap. This situation corresponds to a fluid where all three partial static structure factors are peaked (Fig. I) and the fluid structure resembles that of a metallic glass.

\section{CONCLUSION}


We conclude with two remarks.

(i) In I we neglected the coefficient of thermal diffusion in evaluating the extended hydrodynamic modes of the mixture. This approximation was motivated by the fact that the coefficient of thermal diffusion vanishes in a first-Soninepolynomial approximation, and in the long-wavelength limit it is always much smaller than the diffusion coefficient in mixtures of spheres of not too disparate sizes and masses. ${ }^{1 O}$ On the other hand, in the long-wavelength limit the coefficient of thermal diffusion contributes to the damping of the sound modes. ${ }^{11}$ Since neglecting thermal diffusion is best justified in mixtures of either very low $x_{2}$ or $x_{2} \simeq 1$, one may ask if the absence of the sound-propagation gap at intermediate $x_{2}$ simply occurs because we have underestimated the sound damping by neglecting thermal diffusion. The model used here for the description of the extended sound modes based on the two coupled equations for temperature and longitudinal momentum fluctuations neglects all couplings to the densities and therefore does not contain the coefficient of thermal diffusion. This model still predicts the disappearance and reappearance of the sound propagation gap as a function of concentration, indicating that the dependence of the sound propagation on concentration is not an artifact of the approximation of neglecting thermal diffusion.

(ii) Previous work has focussed on unusual sound propagation in disparate-mass gas mixtures and binary liquid alloys at moderate density. ${ }^{9,12}$ One interesting feature observed in computer simulations ${ }^{13}$ is the appearance of a fast propagating sound mode above a certain nonzero value of the wave vector, signaling an effective separation of the dynamics of light and heavy particles. Campa and Cohen ${ }^{9}$ have shown that the fast sound is a kinetic mode rather than a hydrodynamic mode and it yields an observable shoulder in the dynamic structure factor of gas mixtures. In moderately dense mixtures the fast sound mode is present only for mass ratios below 0.1 and no fast sound occurs for $\eta \geq 0.42$. Our generalized hydrodynamic equations as derived in I cannot yield a fast sound mode since our set of independent variables only includes the conserved densities of the fluid: we only obtain the extended hydrodynamic modes and no kinetic modes. To account for the possibility of fast sound we would need to enlarge our set of independent variables to include at least the relative momentum density and the temperature difference of the two species. On the other hand, the fact that no fast sound is observed in Ref. 9 for the values of the total packing fraction and mass ratio considered here suggests that at such 
high densities the dynamics of light and heavy particles never decouple and the generalized hydrodynamic equations of $\mathbf{I}$ may indeed provide an adequate description of the dynamics at large wave vectors. ${ }^{14}$ A proper test of whether our theory is relevant for real mixtures will, however, only come from a detailed comparison of the dynamic structure factor that can be obtained from the generalized hydrodynamic equations of $\mathbf{I}$ with neutron-scattering spectra from dense mixtures or with numerical simulations. Finally, the fact that the appearance and disappearance of the sound-propagation gap in our work does not depend on the mass ratio and takes place even for equal masses seems to indicate that the fast sound and the concentration dependence of the gap are not related.

\section{ACKNOWLEDGMENTS}

One of us (M.C.M.) thanks T. R. Kirkpatrick for stimulating discussions. This work was supported by the National Science Foundation under Contract No. DMR-8717337.

1 I. M. de Schepper and E. G. D. Cohen, Phys. Rev. A 22, 287 (1980); J. Stat. Phys. 27, 223 (1982).

2 T. R. Kirkpatrick, Phys. Rev. A 32, 3120 (1985).

3 J. R. D. Copley and J. M. Rowe, Phys. Rev. Lett. 32, 49 (1973).

4 A. A. van Well, P. Verkerk, L. A. De Graaf, J. B. Suck, and J. R. D. Copley, Phys. Rev. A 31, 3391 (1985).

${ }^{5}$ I. M. de Schepper, E. G. D. Cohen, and M. J. Zuilhof, Phys. Lett. 103A, 120 (1984).

${ }^{6}$ I. M. de Schepper, J. C. Van Rijs, and E. G. D. Cohen, Physica 1434A, 1 (1985). 
7 M. C. Marchetti and S. Sinha, Phys. Rev. A 41, 3214 (1990).

8 J. B. Suck, H. Rudin, H. J. Guntherodt, and H. Beck, Phys. Rev. Lett. 50, 49 (1983).

9 A. Campa and E. G. D. Cohen, Phys. Rev. A 41, 5451 (1990).

10 J. M. Kincaid, E. G. D. Cohen, and M. Lopez de Haro, J. Chern. Phys. 86, 963 (1987).

11 J. P. Boon and S. Yip, Molecular Hydrodynamics (McGraw-Hill, New York, 1980), pp. 270 and 271.

12 P. B. Lerner and I. M. Sokolov, Physica C 150, 465 (1988).

13 J. Bosse, G. Jacucci, M. Ronchetti, and W. Schirmacher, Phys. Rev. Lett. 57, 3277 (1986).

14 In a one-component hard-sphere fluid with $\eta=0.46$ the generalized hydrodynamic modes, provide a good representation of the dynamic structure factor up to $k \sim 10 / \sigma$, as discussed, for instance, by E. G. D. Cohen, in Trends in Applications of Pure Mathematics to Mechanics, Vol. 249 of Lecture Notes in Physics, edited by E. Kroner and K. Krichgasser (Springer-Verlag, Berlin 1986). 